\title{
Cellular Physiology

\section{C2 Skeletal Myoblast Survival, Death, Proliferation and Differentiation: Regulation by Adra1d}

\author{
Amarjit Saini, Nasser Al-Shanti and Claire Stewart
}

Institute for Biomedical Research into Human Movement and Health, Manchester Metropolitan University, Manchester

\section{Key Words}

Adra1d $\bullet$ TNF- $\alpha \cdot$ IGF-I $\cdot$ Myoblasts $\cdot$ Apoptosis $\cdot$ MAP kinase

\begin{abstract}
IGF-I positively impacts on muscle anabolism/ regeneration. Using $\mathrm{C} 2$ skeletal myoblasts, we previously reported high dose TNF- $\alpha$-induced (10 ng. ml-1) cell death is rescued by IGF-I. However, non-myotoxic low dose TNF- $\alpha\left(1.25 \mathrm{ng} \mathrm{ml}^{-1}\right)$ elicits a MAPK-mediated apoptotic response when coincubated with IGF-I (1.5 ng. $\left.\mathrm{ml}^{-1}\right)$. Our aim was to investigate these conflicting roles of IGF-I in our model. Insulin array and qRT-PCR identified Adra1d as a potential regulatory gene that was up-regulated in survival and down-regulated under apoptotic conditions. TNF- $\alpha$ administration (1.25 or 10 ng. $\mathrm{ml}^{-1}$ ) induced significant decreases $(\sim 50 \%$ both incubations) in Adra1d expression relative to DM. IGF-I addition to high dose TNF- $\alpha$ (10 ng. ml-1) induced myoblast survival and matched a significant $(P<0.05)$ increase in Adra1d expression. By contrast, IGF-I addition to low dose TNF- $\alpha\left(1.25 \mathrm{ng}^{-\mathrm{ml}^{-1}}\right)$ induced elevated death resulting in a significant $(P<0.05)$ decline $(\sim 55 \%)$ in Adra1d expression. Preadministration of PD98059 (20uM), which rescues
\end{abstract}

\section{KARGER}

Fax +4161306 1234

E-Mail karger@karger.ch

www.karger.com
(C) 2010 S. Karger AG, Basel

$1015-8987 / 10 / 0253-0253 \$ 26.00 / 0$

Accessible online at:

www.karger.com/cpb death induced by co-incubation of low dose TNF- $\alpha$ with IGF-I, Adra1d levels were again comparable to DM control. Since Adra1d was elevated following incubations that induced myoblast survival, we investigated effects of Adra1d siRNA gene silencing under these conditions. Adra1d knockdown resulted in significantly higher levels of cell death under all incubations suggesting Adra1d expression is essential for skeletal muscle cell survival.

Copyright () 2010 S. Karger AG, Basel

\section{Introduction}

Prolonged elevations of proinflammatory cytokines (e.g. TNF- $\alpha$ ) are associated with muscle wasting that occurs during the sarcopenia of ageing $[1,2]$ and in several cachectic diseases e.g. AIDS, rheumatoid arthritis and cancer $[3,4]$. Apoptosis represents a potential pathway by which wasting can occur in chronic diseases. While cytokines such as those involved in the TNF- $\alpha$ system act to trigger catabolism it is believed that the IGF system notably IGF-I and IGF-II, positively impacts on muscle anabolism and is essential for skeletal muscle regeneration and maintenance [5]. Clearly, the up-regulation of target

Amarjit Saini

Institute for Biomedical Research into Human Movement and Health

Manchester Metropolitan University, Oxford Road, Manchester, M1 5GD, (UK) Tel. +44 (0)161 247 5713, Fax +44 (0)161 2475751

E-Mail a.saini@mmu.ac.uk 
specific inhibitors of apoptosis, including growth factors and their intracellular signalling pathways, could be highly beneficial in the treatments of muscle wasting disorders.

In mouse models, TNF- $\alpha$ can mimic the cachectic state $[6,7]$ and we confirm from previous studies using the murine $\mathrm{C} 2$ skeletal muscle cell line that TNF- $\alpha$ inhibits myoblast differentiation and leads to cell death $[1,7,8]$. Local levels of the cytokine may therefore have relevance in the pathogenesis of wasting. Because adult skeletal muscle is terminally differentiated, any repair and regeneration of the muscle is dependent on resident satellite or infiltrating stem cells [9] and the ability of these cells to respond to growth and differentiation signals may be dependent on the local growth factor/cytokine environment.

The majority of in vitro studies on skeletal muscle cells have been carried out using immortalised rat and mouse muscle cell lines. These studies have highlighted the importance of the IGFs (IGF-I and IGF-II) in myoblast survival and differentiation $[10,11]$. Binding of IGF-I to its receptor stimulates several downstream signalling events including activation of both mitogen-activated protein kinase (MAPK)/Extracellular regulated kinase (ERK 1/2) and phosphatidylinositol-3-kinase PI(3)K. Both pathways have been implicated in cell growth and differentiation in a variety of cell types. Studies using PI(3)K inhibitors have shown that activation of the PI(3)K signalling pathway is necessary for myoblast differentiation [12]. On the other hand, MAPK (ERK 1/ 2 ) has been implicated in the promotion of murine myoblast proliferation and hence the inhibition of differentiation [13, 14]. Hence, specific activation of key molecules in the IGF signalling pathway is an attractive target for potentially combating muscle wasting by stimulating muscle protein anabolism.

It has been theorised that IGF/TNF interactions are essential for muscle maintenance [15] and our studies have aimed at investigating the impact of exogenous TNF- $\alpha$ and IGF-I on myoblast survival and differentiation. Our findings detailed here have identified that the Adrenergic receptor alpha 1d (Adra1d) may potentially be regulating myoblast survival and differentiation. The $\alpha_{1}$-Adrenergic receptors (AR) mediate many of the physiological functions of the endogenous catecholamines, noradrenaline and adrenaline. The prototypical signalling pathway of $\alpha_{1}$-ARs involves coupling to $G$ proteins of the $\mathrm{G}_{q / 11}$-family $[16,17]$ followed by activation of a phospholipase $\mathrm{C} \beta$ (PLC $\beta)[18,19]$ to yield cleavage of phosphatidylinositol-4,5-bisphosphate into inositol-1,4,5trisphosphate and diacylglycerol $[20,21]$. The former promotes release of $\mathrm{Ca}^{2+}$ from intracellular stores, while the latter activates protein kinase C (PKC). However, $\alpha_{1}$-ARs may also activate a variety of other signalling molecules and are known to constitute a very large family that includes receptors for many peptidic and non-peptidic hormones, drugs and neurotransmitters [22]. Other proximal signalling includes activation of the phospholipases $\mathrm{A}_{2}\left(\mathrm{PLA}_{2}\right)$ via PKC [23] as well as activation of phospholipase D (PLD) [24-26] and elevation of intracellular cAMP concentrations [27]. Numerous additional mediators have been shown or implicated in $\alpha_{1}$-AR signalling, but in most cases it remains to be determined how these, mostly distal events (e.g. cellular hypertrophy, smooth muscle contraction, various ion channels, transporters, protein kinases and transcription factors and proteins related to cell cycle control) relate to the more proximal signalling events mentioned above.

Consequently, with initial array data in this study having revealed a possible role of Adrald in our cells, the aims of the present study were to investigate the potential role of Adra1d in conditions of myotoxic stress and survival induced by TNF- $\alpha$ and IGF-I interactions. We hypothesised that Adra1d is critical for myoblast survival and that cytokine-mediated stress would induce a decline in Adra1d expression whereas conditions of myoblast survival stimulated by growth factor would result in elevated Adra1d expression.

\section{Materials and Methods}

\section{Materials}

All cell and tissue culture media and supplements were purchased as sterile or were filter sterilised through a $0.20 \mu \mathrm{M}$ filter. Heat-inactivated (hi) foetal bovine serum (FBS) and hi new born calf serum (NCS) were purchased from Gibco (Paisley, Scotland); hi horse serum (HS) from TCS Biosciences (Corby, England); penstrep (penicillin and streptomycin) and trypsin from Bio Whittaker (Wokingham, England); L-glutamine from BDH (Poole, England), gelatine from Sigma (St. Louis, U.S.A.). Glassware, distilled water $\left(\mathrm{dH}_{2} \mathrm{O}\right)$ and phosphate buffered saline (PBS, Oxoid (Hampshire, England)) were autoclaved prior to use. Sterile media were stored at $4^{\circ} \mathrm{C}$ and used within 2 months. Plasticware were purchased as sterile from Greiner Bio-one, (Kremsmunster, Austria) unless otherwise stated. Recombinant human TNF- $\alpha$ and PD98059 were purchased from Calbiochem (Nottingham, England); TRIZOL reagent (Invitrogen, Life Technologies), propidium iodide (PI) stain from BD Biosciences (San Diego, U.S.A.) and creatine kinase assay kits from Catachem (Bridgeport, U.S.A.). Recombinant human IGF-I as purchased from GroPep (Adelaide, Australia). BCA protein quantitation reagents were obtained from Pierce Chemical (Chester, England). 


\section{Cell culture}

C2 mouse skeletal myoblasts [28] were grown in a humidified $5 \% \mathrm{CO}_{2}$ atmosphere at $37^{\circ} \mathrm{C}$ in growth medium (GM), composed of: DMEM with Glutamax supplemented with $10 \%$ FBS, $10 \%$ NCS, penstrep and l-glutamine, at final concentrations of $10000{\mathrm{U} . \mathrm{ml}^{-1}}^{-1}$ and $2 \mathrm{mM}$ respectively, until $80 \%$ confluency was attained. Experiments and differentiation were initiated following washing with phosphate buffered saline (PBS), by transferring to low serum-containing differentiation medium (DM; DMEM plus glutamax, supplemented with 2\% HS, penstrep and L-glutamine (supplemented as above)) in the absence or presence of specific cytokines, growth factors and signal inhibitors. C2 myoblasts are able to undergo spontaneous differentiation into myotubes on serum withdrawal, and do not require growth factor addition to stimulate the process [10]. Adherent cells following trypsinisation, and detached cells in the supernatant, were counted using a haemocytometer in the presence of trypan blue dye (Bio Whittaker, Wokingham, England).

\section{Cell treatments}

Six-well plates were pre-coated with $0.2 \%$ gelatine for 5 minutes at room temperature. Following removal of excess

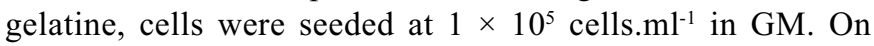
attaining approximately $80 \%$ confluency, cells were washed twice with PBS and transferred to DM for 30 minutes at $37^{\circ} \mathrm{C}$. The end of this 30 minute equilibration time-point was denoted time 0 .

\section{Interactions of $T N F-\alpha$ and $I G F-I$}

To assess the impact of cytokine and growth factor interactions on myoblast survival or death; at time 0 myoblasts received vehicle or TNF- $\alpha$ (1.25 or $\left.10 \mathrm{ng}^{-1} \mathrm{ml}^{-1}\right)$. After $1 \mathrm{~h}$ the cytokine pre-incubated myoblasts received IGF-I (1.5 ng. $\left.\mathrm{ml}^{-1}\right)$, for appropriate times up to 72-h.

Following 36-h incubation, RNA was extracted from myoblasts for RNA arrays and for real time RT-PCR. For cell counting and flow cytometric studies, cells were trypsinised $48 \mathrm{~h}$ following dosing. For studies involving creatine kinase assays, cells were harvested following $72-\mathrm{h}$ incubation, as described below.

\section{TNF- $\alpha$ and IGF-I inhibitor studies}

Where inhibitor studies were performed, upon reaching $80 \%$ confluency, cells were washed twice in PBS and transferred to $\mathrm{DM}$ for $30 \mathrm{~min}$ in the absence or presence of the MEK inhibitor, PD98059 $(20 \mu \mathrm{M})$ or PI(3)K inhibitors LY204002 $(5 \mu \mathrm{M})$ for 30 minutes at $37^{\circ} \mathrm{C}$. The end of this $30 \mathrm{~min}$ incubation was designated time 0 . At time 0 (either with or without inhibitor) cultures were subsequently spiked with TNF- $\alpha$ (1.25 or $10 \mathrm{ng}$. $\left.\mathrm{ml}^{-1}\right)$ followed $1 \mathrm{~h}$ later with IGF-I $\left(1.5 \mathrm{ng} \cdot \mathrm{ml}^{-1}\right)$ as described above.

\section{Insulin RNA array}

RNA array was performed using the Oligo GEArray SuperArray kits from SuperArray Bioscience (P.A., U.S.A.) containing 128 oligonucleotide probes that represented genes associated with the insulin signalling pathway (Mouse insulin

Role of Adra1d in TNF- $\alpha$ and IGF-I Iteractions microarray signalling pathway OMM-030). In brief RNA hybridisation was carried out according to the manufacturer's instructions. $3.5 \mu \mathrm{g}$ of biotin-labelled cRNA prepared using the TrueLabelling-AMP $2.0 \mathrm{Kit}$ (GA-030) was added to $0.75 \mathrm{ml}$ of pre-warmed GEAhyb Hybridisation Solution. This was mixed and hybridised overnight in a hybridisation oven (Problot 12S, Labnet, Oakhum, Rutland, U.K.) at $60^{\circ} \mathrm{C}$ with continuous agitation (5 to $10 \mathrm{rpm}$ ). The membranes were washed and visualised using a chemiluminescent detection kit (D-01) from SuperArray Bioscience (P.A., U.S.A.). Images were captured using the BioRad imaging system and were analysed (measure the intensity of chemiluminescence of each square grid representing a gene) using GEArray Expression Analysis Suite by Bioscience, MD, U.S.A.

\section{Primer design and synthesis}

We used web-based software from Invitrogen to design our primers which were further analysed by Sigma-Genosys software. The primers for the mouse Adrald gene (Reference sequence no. NM_013460) F: TTCGTCCTGTGCTGGTTC; R: GGGTAGATGAGCGGGTTC were designed to yield products spanning exon-intron boundaries to prevent any possible genomic DNA-contaminations from total RNA isolation. Sequence homology searches against the GenBank database showed that these primers matched only the sequence to which they were designed. Primers were synthesised by SigmaGenosys (Suffolk, UK) which are compatible with real-time PCR without further purification

\section{$R N A$ extraction and $q R T-P C R$}

Following cell treatments, cells were washed with PBS and lysed with $200 \mu 1$ TRIZOL reagent. RNA was extracted from TRIzol homogenates according to the manufacturer's instructions (Invitrogen, CA). The iScript ${ }^{\mathrm{TM}}$ One-Step RT-PCR Kit with SYBR Green, was used to perform qRT-PCR. 30ng of RNA was used per qRT-PCR reaction which was performed with the Chrom4 Detection System from Biorad laboratories (Hercules, CA, U.S.A.). Opticon Monitor Version 3.1 software from Biorad Laboratories (Hercules, CA, U.S.A.) was used to analyse qRT-PCR data.

The relative gene expression levels were calculated using the comparative $\mathrm{Ct}\left({ }^{\Delta \Delta} C_{\mathrm{t}}\right)$ method (described in the Bio-rad qRTPCR application guide), where the relative expression is calculated as $2^{-\Delta \Delta \mathrm{Ct}}$ and where $C_{\mathrm{t}}$ represents the threshold cycle. Experiments were run 3 times in duplicate.

\section{siRNA design and transfection}

siRNA molecules used for silencing murine Adrald were designed for the mouse Adrald gene (Genbank Accession No. NM 013460) using the Whitehead Institute for Biomedical Research siRNA Selection Programme (http://jura.wi.mit.edu/ bioc/siRNAext/) based design parameters developed by Tuschl and co-workers [29-32]. Searches of the genome database (BLAST, SSEARCH) were carried out to ensure that these sequences would not target any other murine genes. The siRNA were synthesised by Ambion, Applied Biosystems. The most effective siRNA dose at silencing the target-gene was determined by carrying out dose response $(0-80 \mathrm{nM})$ studies. 
Fig. 1. Apoptotic Interaction of IGF-I and TNF- $\alpha$. Myoblast death is presented as percentage of positive cells in the preG1 phase of the cell cycle (A). Myoblasts pre-administered with a MAPK inhibitor prevent the apoptotic potential of low dose co-incubated TNF- $\alpha$ and IGF-I, but not high dose TNF- $\alpha$ (B). Myoblasts were initially transferred into differentiation medium alone or supplemented with $20 \mu \mathrm{M}$ PD98059 for 30 -minutes before being administered with TNF- $\alpha$ or IGF-I alone or in combination (1.25 or 10 ng. $\mathrm{ml}^{-1}$ TNF- $\alpha$ pre-incubation then $\left.1.5 \mathrm{ng} \cdot \mathrm{ml}^{-1} \mathrm{IGF}-\mathrm{I}\right)$.

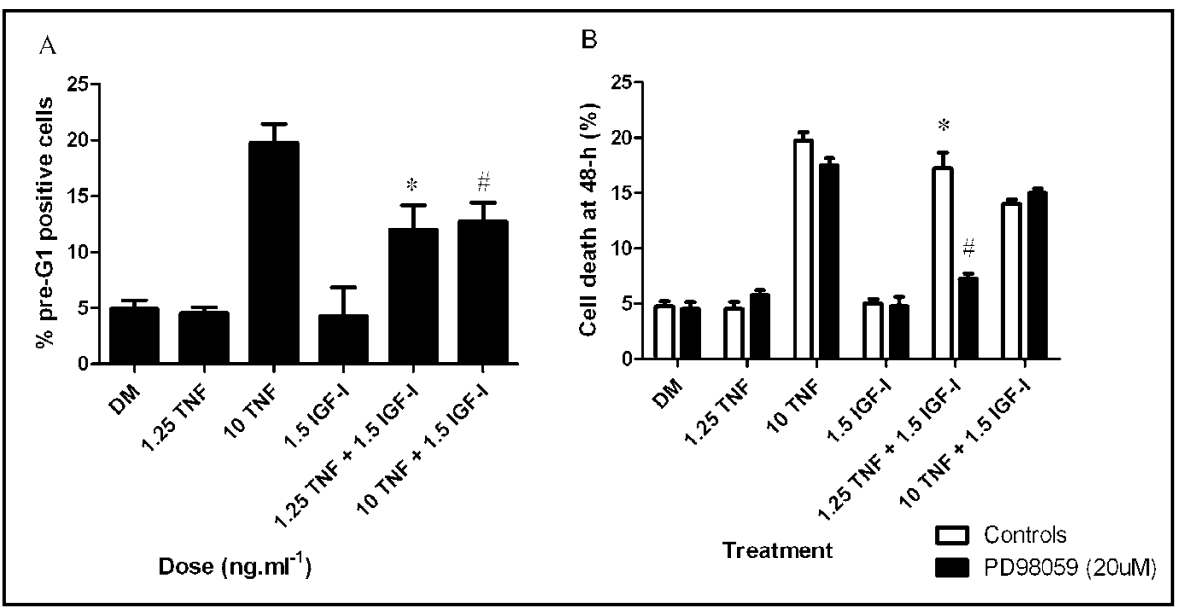
Cells were incubated for $48 \mathrm{hrs}$. Data are presented as Mean \pm SEM $\left(\mathrm{n}=3\right.$, in duplicate for each group). ${ }^{*} \mathrm{P}<0.05$ significantly different from DM, \# 10 ng.ml ${ }^{-1}$ TNF- $\alpha$ (A) or \# 1.25 ng.ml ${ }^{-1}$ TNF- $\alpha$ and 1.5 ng.ml-1 IGF-I control (B).

Transfections with siRNA were performed according to the manufacturer's instructions (siPORT NeoFX reagent, Ambion, Applied Biosystemns, TX, USA). In each experiment, negative siRNA (Silencer Negative Control siRNA (40 nM) (Applied Biosystemns, UK) which has no significant homology to any known gene sequences and medium alone were included as negative controls whereas Silencer siRNA for GAPDH (40 nM - reduces the expression of GAPDH by 70-95\%) (Applied Biosystemns, UK) was included as a positive control. After 36-h, RNA was extracted from transfected cells and gene inhibition determined using qRT-PCR. All optimisation experiments were performed at least twice in duplicate.

\section{siRNA optimisation}

Adrald siRNA was synthesised by Ambion, Applied Biosystems, UK. Dose response studies determined Adra1d siRNA sequence - F: GCCAUUAUGACAGAACGCATT; R: UGCGUUCUGUCAUAAUGGCTG - at $40 \mathrm{nM}$ to induce $66 \pm 5 \%$ gene inhibition (data not shown).

\section{Analysis of cell cycle by flow cytometry}

After 48-h incubation, adherent cells were trypsinised in $0.2 \mathrm{ml}$ of $0.5 \%$ trypsin $/ 0.02 \%$ EDTA solution and were pooled with detached cells, resuspended and washed in PBS prior to fixing at $-20^{\circ} \mathrm{C}$ in $70 \%$ ethanol. After a minimum of $24-\mathrm{h}$, cells were pelleted $(200 \times \mathrm{g}$ for 5 -min), washed in PBS (three times $200 \times \mathrm{g}$ for 5-min) and resuspended with gentle vortexing in propidium iodide labelling buffer $\left(50 \mu \mathrm{g} . \mathrm{ml}^{-1}\right.$ propidium iodide, $0.1 \%$ sodium citrate, $20 \mu \mathrm{g} . \mathrm{ml}^{-1}$ ribonuclease A, $0.3 \%$ Nonidet $\mathrm{P}-40, \mathrm{pH} 8.3$ ) at approximately $\sim 1 \times 10^{6}$ cells.ml ${ }^{-1}$. Cells were stored in the dark at $4{ }^{\circ} \mathrm{C}$ for 30 -min, prior to assaying at room temperature, using a Becton Dickinson FACSCalibur flow cytometer. Data were analysed using Cell Quest (Becton Dickinson, Oxford, England).

\section{Creatine kinase assay}

Cells were treated as described in sections 2.3-2.5, washed twice with PBS and lysed in $0.2 \mathrm{ml}$ of $50 \mathrm{mM}$ Tris-MES, $\mathrm{pH} 7.8$, $1 \%$ Triton X-100 (TMT buffer). Samples were stored at $-80^{\circ} \mathrm{C}$, and assayed within 2 weeks of harvesting using a commercially available kit (Catachem CK assay) according to manufacturers instructions. Enzymatic activity was normalised to total protein content as determined by the $\mathrm{BCA}^{\mathrm{TM}}$ assay (Pierce, Rockford, IL, U.S.A.).

\section{Statistical analysis}

Data were analysed using Microsoft Excel version 7.0 and SPSS version 11.5 software. Results are presented as the mean \pm standard error of the mean (SEM). Statistical significance was determined using one-way analysis of variance with multiple post hoc analyses. Results were considered statistically significant when the $\mathrm{P}$ value was $<0.05$. All experiments were performed at least 3 times in duplicate, unless otherwise stated.

\section{Results}

\section{Effects of TNF- $\alpha$ and IGF-I on skeletal myoblast death and differentiation}

We have previously demonstrated that $\mathrm{C} 2$ skeletal myoblasts are susceptible to apoptotic death, in a dose responsive fashion to TNF- $\alpha[1,15]$. Basal myoblast death $(\leq 5 \%)$ was significantly $(\mathrm{P}<0.05)$ increased at doses of $2.5,5,10$ and plateauing at $20 \mathrm{ng} \cdot \mathrm{ml}^{-1}$. At $10 \mathrm{ng} \cdot \mathrm{ml}^{-1}$, TNF- $\alpha$ induced a significant 16 -fold increase in myoblast death, which was confirmed to be apoptotic using flow cytometry (Figure 1A). Critically, the capacity of the cells to differentiate and to form myotubes was also blocked $(\mathrm{P}<0.05)$ at all doses of TNF- $\alpha$ tested, even those which did not induce apoptosis $\left(1.25 \mathrm{ng} \cdot \mathrm{ml}^{-1}\right)$ [1]. Furthermore, although low dose IGF-I ( $\left.1.5 \mathrm{ng} \cdot \mathrm{ml}^{-1}\right)$ was able to reduce the apoptotic effects of high dose TNF- $\alpha\left(10 \mathrm{ng}^{\mathrm{ml}} \mathrm{l}^{-1}\right)$, it was not able to rescue the block on differentiation.

Similarly, myotube formation and creatine kinase activity were blocked by 1.25 ng. $\mathrm{ml}^{-1} \mathrm{TNF}-\alpha$ and again, 
to our surprise, IGF-I regardless of dose was unable to

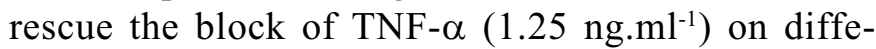
rentiation [1]. In contrast to relieving the block on differentiation, co-incubation of low dose TNF- $\alpha$, which did not induce cell death, compared with controls, when administered with low dose IGF-I ( $\left.1.5 \mathrm{ng}^{-\mathrm{ml}^{-1}}\right)$ culminated in cell death. To confirm that death was by apoptosis in the presence of low dose TNF- $\alpha$ (1.25 ng. $\left.\mathrm{ml}^{-1}\right)$ and IGF-I $\left(1.5 \mathrm{ng} \cdot \mathrm{ml}^{-1}\right)$ flow cytometry was performed and demonstrated that while triggers alone were without effect on death, a significant doubling in pre-G1 positive cells in the presence of low dose TNF- $\alpha\left(1.25 \mathrm{ng}^{-\mathrm{ml}^{-1}}\right)$ and IGFI (1.5 ng. $\left.\mathrm{ml}^{-1}\right)$ compared with DM or either trigger alone (Figure 1A). This increase elicited by a co-incubation of low dose TNF- $\alpha$ (1.25 ng. $\left.\mathrm{ml}^{-1}\right)$ plus IGF-I (1.5 ng. $\left.\mathrm{ml}^{-1}\right)$ was blocked $(\mathrm{P}<0.05)$ by a pre-incubation with PD98059 $(20 \mu \mathrm{M}$; Figure 1B). In contrast, high dose TNF- $\alpha(10$ ng. $\left.\mathrm{ml}^{-1}\right)$ induced death is unaffected by PD98059 $(20 \mu \mathrm{M}$; Figure 1B) as no significant changes were observed in the presence of the MAPK inhibitor and relies on alternative pathways already demonstrated by Stewart and coworkers [33].

\section{Gene expression in TNF- $\alpha$ and IGF-I cultured myoblasts}

Having determined that high dose TNF- $\alpha$ (10 ng. $\mathrm{ml}^{-1}$ ) induces apoptosis which is rescued by IGF-I and low dose TNF- $\alpha$ (1.25 ng. $\left.\mathrm{ml}^{-1}\right)$ becomes apoptotic in the presence of IGF-I we wished to examine the potential mechanisms of these differential responses. To this end we performed gene array technology and qRT-PCR for insulin/survival pathways to examine the contrast in responses associated with low and higher doses of TNF- $\alpha$ and their interaction with IGF-I. Analysis of the insulin arrays for control incubations (Figure 2A) shows that only DM $(\mathrm{n}=10$ genes $)$, IGF-I $\left(1.5 \mathrm{ng} \cdot \mathrm{ml}^{-1}\right)$ $(\mathrm{n}=5$ genes $)$ and low dose TNF- $\alpha\left(1.25 \mathrm{ng}^{-\mathrm{ml}^{-1}}\right)(\mathrm{n}=5$ genes) alone evoked $\mathrm{a} \geq 2$-fold increase in insulin response gene expression. Importantly, there was no overlap in expression of upregulated genes in the presence of IGF-I or low dose TNF- $\alpha$. With administration of high dose TNF- $\alpha$ (10 ng. $\left.\mathrm{ml}^{-1}\right)$ no insulin genes were upregulated, indeed the majority of genes $(n=65)$ fell below the 0.5 expression level, suggesting gene suppression. With the addition of IGF-I to high dose TNF$\alpha\left(10\right.$ ng. $\left.\mathrm{ml}^{-1}\right)$ which induces survival $\mathrm{n}=7$ insulin genes were up-regulated by $>2$-fold (Figure 2B). This increase in gene expression was not observed when IGF-I was co-incubated with low-dose TNF- $\alpha$ when all genes were suppressed.

Role of Adra1d in TNF- $\alpha$ and IGF-I Iteractions

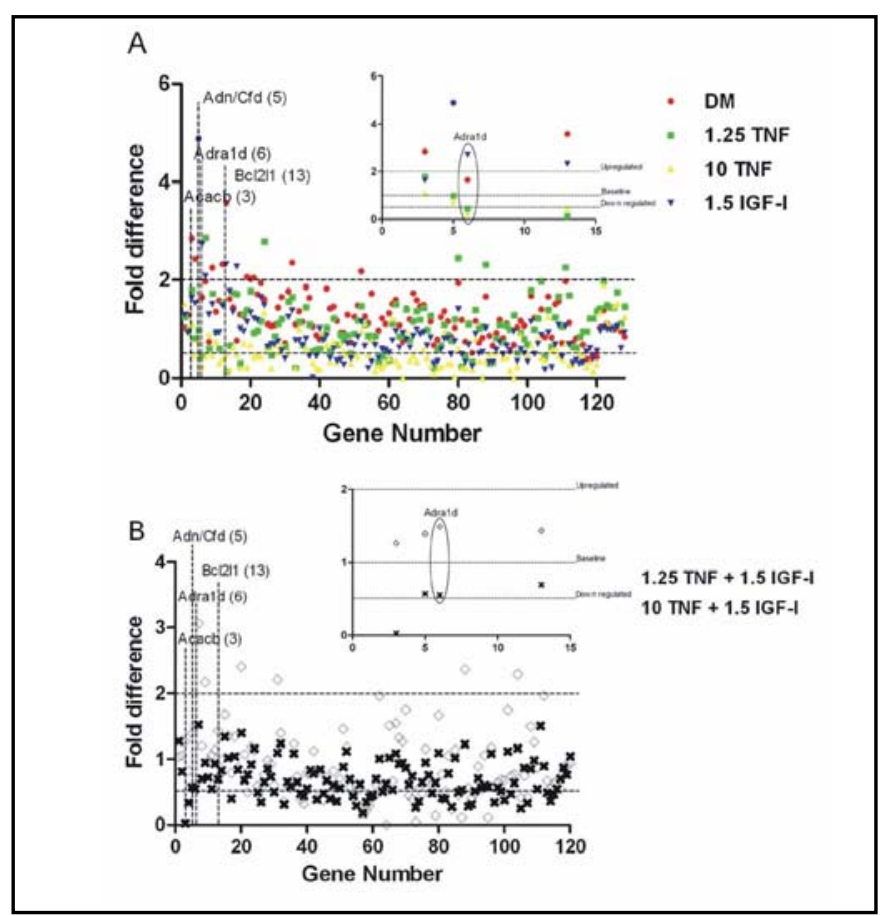

Fig. 2. Gene array analysis of insulin signalling pathway. Following $36-\mathrm{h}$ with TNF- $\alpha$ (1.25 or $\left.10 \mathrm{ng} \cdot \mathrm{ml}^{-1}\right)$ or IGF-I (1.5 ng. $\mathrm{ml}^{-1}$ ) alone (A) or as co-incubations (B) RNA was extracted from myoblasts to create purified biotin-labelled cRNA and hybridised onto insulin array membranes. Subsequently membranes were visualised using chemiluminescence and emissions recorded using BioRad imaging system to measure gene expression levels for 128 genes (Mouse insulin microarray signalling pathway OMM-030) relative to control myoblasts transferred from growth medium into low serum medium for 30 minutes and denoted time point 0 (T0). Data are presented as Mean ( $\mathrm{n}=2$, in duplicate for each group).

\section{Expression of survival genes in TNF- $\alpha$ and IGF-I cultured myoblasts}

Wishing to confirm the array data, we performed specific qRT-PCR studies. The genes selected for further analyses, Acacb, Adn/Cfd, Bcl211 and Adrald were those which increased in the presence of DM or IGF-I where greatest myoblast survival rates were observed but were decreased by TNF- $\alpha\left(10 \mathrm{ng}^{-\mathrm{ml}^{-1}}\right)$ or low TNF- $\alpha$ (1.25 ng. $\mathrm{ml}^{-1}$ /IGF-I where greatest myoblast death was reported. Of the genes selected qRT-PCr, Adrald showed the most notable and confirmatory relative differences in gene expression and was therefore selected for further analyses. Administration of TNF- $\alpha$ (1.25 or 10 ng.ml $\left.{ }^{-1}\right)$ induced a significant $(\mathrm{P}<0.05)$ decrease in Adrald ( $\sim 50 \%$ in both incubations) compared to control DM, thus matching the array. IGF-I alone resulted in a significant $(\mathrm{P}<0.05) 30 \%$ increase in Adra1d expression levels, again in line with the array data (Figure 3A). If Adrald is important for survival then expression should increase 


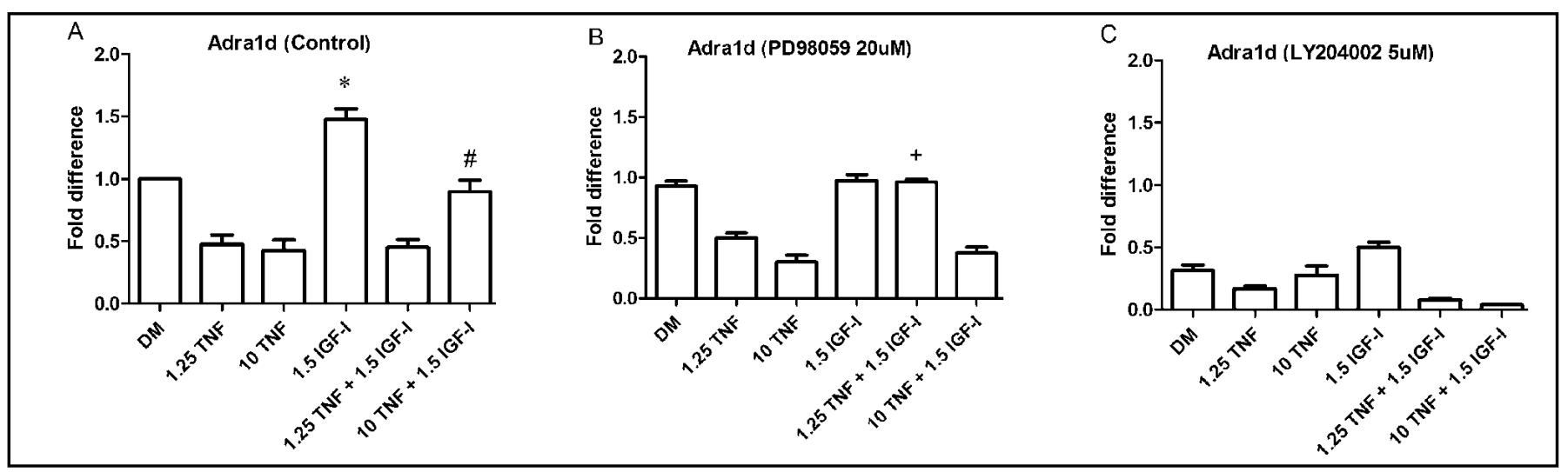

Fig. 3. Interaction of myoblast apoptosis/survival with Adrald expression in the presence or absence of MAPK specific inhibitor PD98059 and PI(3)K specific inhibitor LY204002. qRT-PCR was performed using Biorad (iScript ${ }^{\mathrm{TM}}$ One-Step RT-PCR Kit with SYBR Green) for Adra1d expression. Gene expression levels were calculated relative to DM control using the comparative Ct $\left(\Delta \Delta C_{\mathrm{t}}\right)$ method. Data are presented as Mean $\pm \mathrm{SEM}\left(\mathrm{n}=4\right.$, in duplicate for each group). ${ }^{*} \mathrm{P}<0.05$ significantly different from all incubations; \# (10 ng. $\mathrm{ml}^{-1}$ TNF- $\alpha$ co-incubated with $1.5 \mathrm{ng} \cdot \mathrm{ml}^{-1} \mathrm{IGF}-\mathrm{I}$, control) $\mathrm{P}<0.05$ significantly different from $10 \mathrm{ng} \cdot \mathrm{ml}^{-1}$ alone, control; + (1.25 ng. $\mathrm{ml}^{-1}$ TNF- $\alpha$ co-incubated with 1.5 ng. $\mathrm{ml}^{-1}$ IGF-I, PD98059 pre-incubation) $\mathrm{P}<0.05$ significantly different from myoblasts pre-administered $20 \mu \mathrm{M}$ PD98059 1.25 ng.ml ${ }^{-1}$ TNF- $\alpha$ alone.

when $10 \mathrm{ng} \cdot \mathrm{ml}^{-1}$ of TNF- $\alpha$ is co-incubated with IGF-I (1.5 ng. $\left.\mathrm{ml}^{-1}\right)$ compared with TNF- $\alpha\left(10\right.$ ng. $\left.\mathrm{ml}^{-1}\right)$ alone. This is indeed the case (Figure $3 \mathrm{~A}$ ). In contrast, to high dose TNF- $\alpha$, the addition of IGF-I to low dose TNF- $\alpha$ $\left(1.25 \mathrm{ng} . \mathrm{ml}^{-1}\right)$ resulted in an increase in death. Consequently, it was expected that Adrald expression would remain down-regulated under these conditions, as was the case (Figure 3A). Notably the changes in Adrald in the presence of IGF-I (increase) or low dose TNF (decrease), both of which influence differentiation in a positive or negative manner, respectively, suggest Adrald may also influence myotube formation.

\section{Impact of MAPKK or PI(3)K inhibition on Adrald} expression

Having investigated the impact of the interaction of TNF with IGF on survival, death and Adrald expression and having acquired data to show that not only is Adra1d expression significantly increased under survival and differentation conditions, but that its levels decline when cell death is elevated (low dose TNF- $\alpha$ co-incubated with IGF-I and high dose TNF- $\alpha$ alone), and differentiation is blocked (low dose TNF- $\alpha$ ) we next wished to investigate whether the reduction of death in myoblasts co-incubated with low dose TNF- $\alpha$ /IGF-I pre-administered with PD98059 would also be associated with a up-regulation in Adrald expression. Indeed, in the presence of PD98059 $(20 \mu \mathrm{M})$ which has no effect on basal TNF- $\alpha$ or IGF-I induced death the most notable difference was observed following co-incubation of low dose TNF- $\alpha$ (1.25 ng. $\left.\mathrm{ml}^{-1}\right)$ with IGF-I (1.5 ng. $\mathrm{ml}^{-1}$ ) (Figure 3B), where these co-incubation conditions caused a reduction in Adrald expression, which was reversed in the presence of the MAPKK inhibitor, which also evoked cell rescue (Figure 3B). Importantly, under conditions where death is blocked, and Adrald expression returns to baseline, differentiation is still not rescued over the 72 hour time frame suggesting a more potent pro-survival than pro-differentiation role of this agent during the duration of our studies.

We further investigated regulation of this gene and assessed the impact of PI(3)K inhibition on its expression (Figure 3C). Inhibition of the PI(3)K pathway, using LY204002 $(5 \mu \mathrm{M})$ resulted in the lowest recorded Adrald expression for all treatments investigated, including incubation of DM or IGF-I alone (1.5 ng. $\left.\mathrm{ml}^{-1}\right)$ and in both co-incubated conditions for low and high dose TNF- $\alpha$ $\left(1.25 / 10 \mathrm{ng}^{-1} \mathrm{ml}^{-1}\right)$. The inhibition of the $\mathrm{PI}(3) \mathrm{K}$ pathway was also associated with significantly $(\mathrm{P}<0.05)$ increased incidence of myoblast death only in high dose TNF- $\alpha$ / IGF-I co-incubations (from $12 \pm 1 \%$ to $21 \pm 3 \%$; data not shown however Adrad suppression by LY204002 was associated with a block in differentiation under all conditions tested. These data suggest that the PI(3)K pathway is generally essential for regulating Adra1d expression and differentiation, although under basal conditions when metabolism is reduced (in the presence of LY294002), a resultant reduction in Adrald is not associated with increased cell death. 
Fig. 4. The effects of Adrald inhibition on myoblast survival. Following preadministration with Adrald siRNA or Negative siRNA control for 24-h in GM myoblasts were transferred into differentiation medium alone and/or dosed with $1.5 \mathrm{ng} . \mathrm{ml}^{-1} \mathrm{IGF}-\mathrm{I}$ or co-incubated with $1.25 \mathrm{ng} . \mathrm{ml}^{-1}$ and $1.5 \mathrm{ng} \cdot \mathrm{ml}^{-1}$ IGF-I preadministered with $20 \mu \mathrm{M}$ MAPK blocking agent (PD98059). Representative photomicrographs at 48 -h of random fields from $n=3$ independent experiments in duplicate (A). Myoblasts were stained with trypan blue and damage quantified as percentage death from $n=3$ independent experiments in duplicate (B). Flow cytometric analysis. Samples were fixed in $75 \%$ ethanol and resuspended with propidium iodide. Data are representative of $n=3$ duplicate samples (C). All data are presented as Mean \pm SEM. $* \mathrm{P}<0.05$ significantly different from all incubations. *a $\mathrm{P}<0.05$ significantly different from negative siRNA control DM.

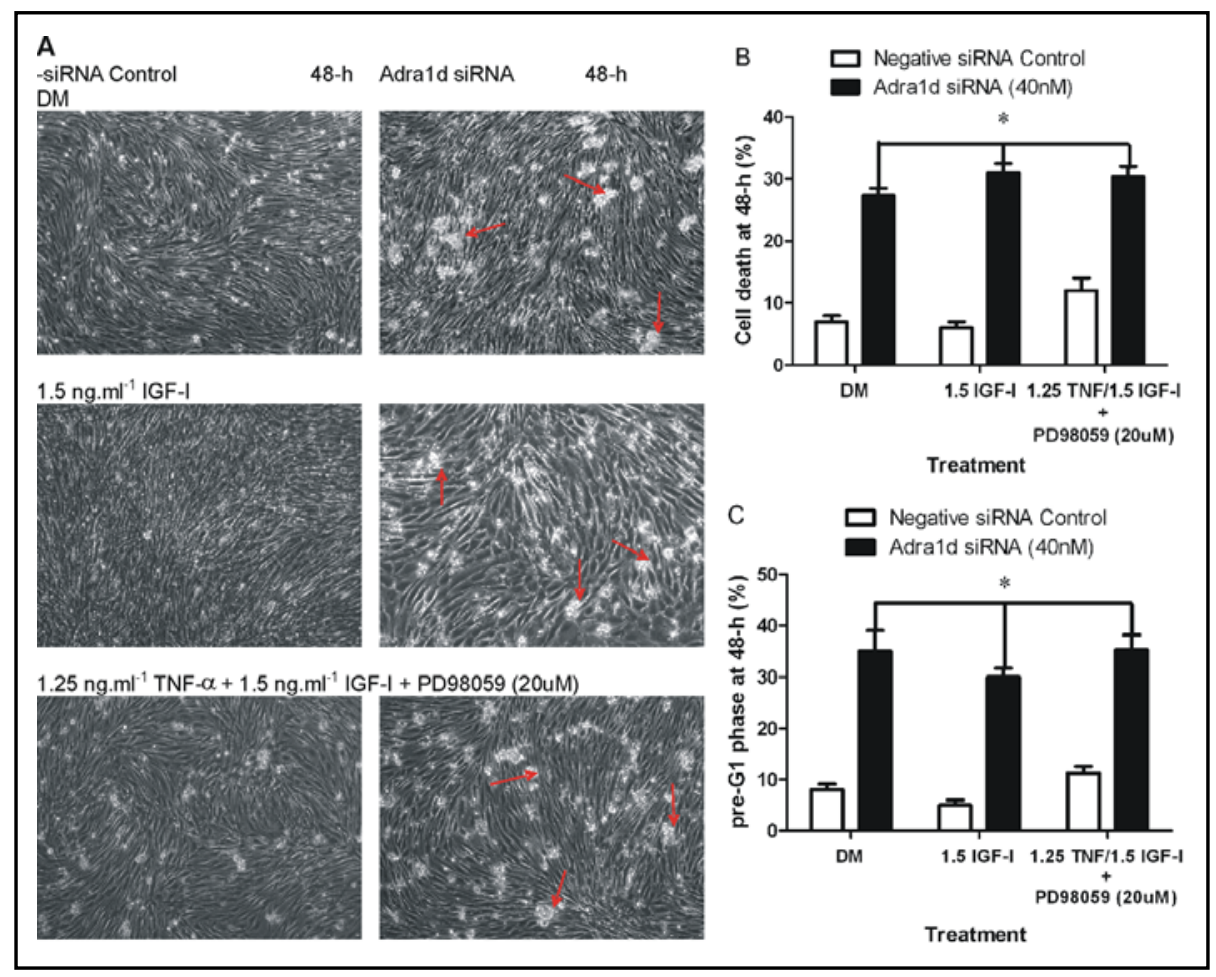

Effect of Adrald inhibition on skeletal myoblast death and differentiation

Adrald expression appeared to be associated with survival mechanisms in metabolically active, stressed cells. We therefore wished to ascertain, using siRNA technology, whether Adra1d was simply a driver of prosurvival mechanisms under situations of stress or whether its expression was essential for the normal metabolic health (in the presence of an active PI(3)K pathway) of the myoblasts. Preliminary dose response experiments, to ascertain working concentrations of siRNA identified that 40nM Adra1d siRNA was effective at inhibiting $66 \pm 5 \%$ of Adra1d expression under basal conditions and in myoblasts treated with IGF-I alone $\left(1.5 \mathrm{ng} \cdot \mathrm{ml}^{-1}\right)$ or with co-incubations of low dose TNF- $\alpha\left(1.25 \mathrm{ng}^{\left.-\mathrm{ml}^{-1}\right) / \mathrm{IGF}-\mathrm{I}}\right.$ co-incubation in the presence of MAPK inhibitor PD98059 (20uM) (data not shown), situations where Adra1d levels were previously found to be enhanced or at basal levels, respectively.

Morphological data at 48-h (Figure 4A), demonstrated that when Adrald was inhibited in DM, IGF-I (1.5 ng. $\left.\mathrm{ml}^{-1}\right)$ and low dose TNF- $\alpha\left(1.25\right.$ ng. $\left.\mathrm{ml}^{-1}\right) /$ IGF-I (1.5 ng.ml $\left.\mathrm{l}^{-1}\right)$ plus PD98059 (20 uM) conditions, higher levels of cell detachment and death were evident compared with their counterparts receiving control siRNA transfection. Wishing to confirm the morphological data, cell death measured by trypan blue exclusion counts was calculated (Figure 4B), and flow cytometric analyses were

Role of Adra1d in TNF- $\alpha$ and IGF-I Iteractions performed to confirm that death was by apoptosis (Figure 4C). Confirming morphological data (Figure 4A), Figure 4B shows a significant $(\mathrm{P}<0.05) 3-4$ fold increase in myoblast death following inhibition of Adrald expression relative to siRNA Control counterparts regardless of the incubation conditions. Total cell number was unchanged (data not shown). Death was confirmed to be apoptotic by flow cytometry where again there was an $\sim 4$-fold significant $(\mathrm{P}<0.05)$ increase in death following Adra1d suppression, regardless of the culture conditions (Figure 4C). Importantly, the increase in apoptotic death in myoblasts administered with the Adrald siRNA in the presence of DM and IGF-I (1.5 ng. $\left.\mathrm{ml}^{-1}\right)$, but absence of PI(3)K inhibition, suggests that in our cell model, Adrald expression is essential for basal cell survival.

\section{Adrald silencing inhibits differentiation}

Having monitored the effect of Adrald silencing on cell death and having established its crucial role in myoblast survival, we next investigated its impact on differentiation. Creatine kinase activity, a biochemical marker of myoblast differentiation and consequent myotube formation was apparent after 72-h only in siRNA Control transfected samples administered with DM or IGF-I alone (Figure 5A). In myoblasts administered with Adrald siRNA, creatine kinase activity remained at basal levels and was associated with a lack of myotube formation. Most striking was the effect of Adrald inhibition on cell morphology at 
Fig. 5. The effect of Adrald inhibition on myotube formation. Following pre-administration with Adrald siRNA or Negative siRNA Control for 24-h in GM myoblasts were transferred into differentiation medium alone and/or dosed with $1.5 \mathrm{ng} . \mathrm{ml}^{-1}$ IGF-I or co-incubated with $1.25 \mathrm{ng} \cdot \mathrm{ml}^{-1}$ and $1.5 \mathrm{ng} \cdot \mathrm{ml}^{-1} \mathrm{IGF}-$ I pre-administered with $20 \mu \mathrm{M}$ MAPK blocking agent (PD98059). Creatine kinase activity to assess myofibre formation from $\mathrm{n}=3$ independent experiments in duplicate (A). Images were assessed at $\times 100$ magnification. Representative photomicrographs of random fields from $n=3$ independent experiments in duplicate (B). Supernatant which contained the damaged/suspended myoblasts were stained with trypan blue and damage of myoblasts in suspension was quantified as percentage death from $n=3$ inde-

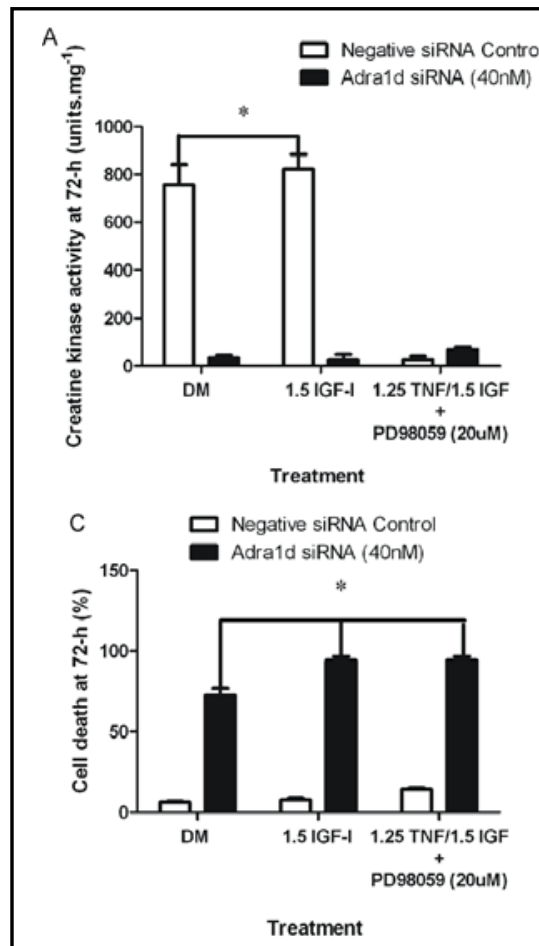

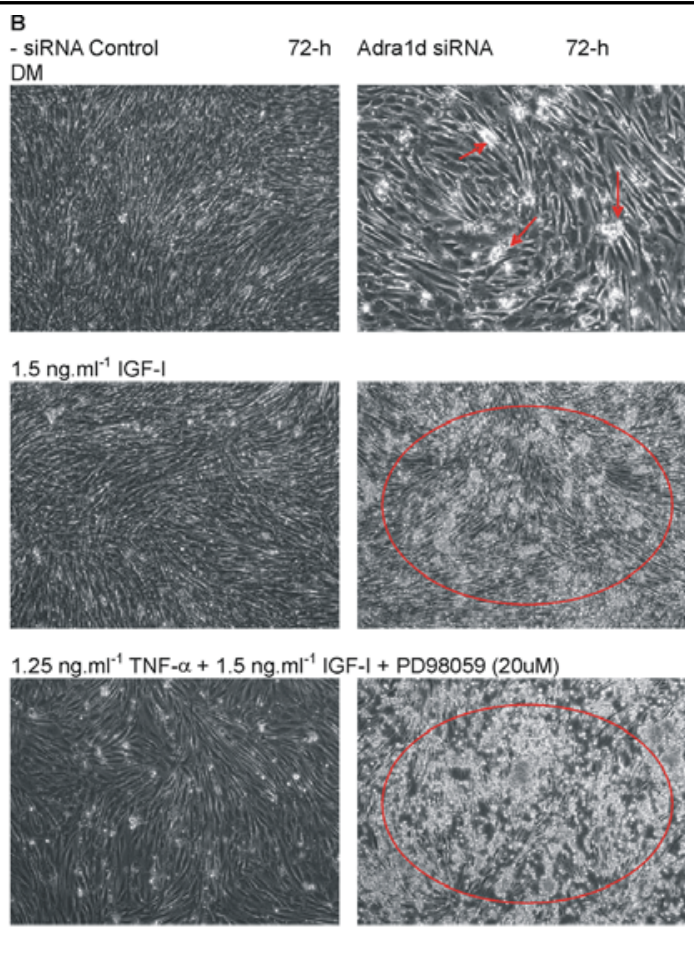

pendent experiments in duplicate $(\mathrm{C})$. All data are presented as Mean $\pm \mathrm{SEM}$. ${ }^{*} \mathrm{P}<0.05$ significantly different from all incubations. 72-h. Cell detachment was widespread for each dose relative to siRNA control (Figure 5B) and analyses of the supernatant indicated that the majority of the test myoblasts were dead (Figure 5C). These data suggest in the absence of Adrald, death is progressive over time (from 48 to $72-h$ ) and myoblasts struggle to survive.

\section{Discussion}

\section{Insulin and survival signalling in TNF- $\alpha$ and} IGF-I cultured myoblasts

While the precise concentrations of TNF- $\alpha$ in skeletal muscle with age and disease are not well documented, cell culture studies on differentiated skeletal muscle myotubes using clinically relevant systemic doses of TNF- $\alpha$ (1-6 ng. $\mathrm{ml}^{-1}$ have been reported in serum from cancer and cachectic patients) [34] have shown that exposure (1-3 ng.ml ${ }^{-1} \mathrm{TNF}-\alpha$ ) results in muscle protein degradation $[34,35]$. Concurrently, observations made in vivo in a rodent model (100 ng. $\mathrm{kg}^{-1}$ bodyweight) reported increased nitrogen and amino acid efflux from skeletal muscle and loss of body protein [36, 37]. Very low concentrations of TNF- $\alpha\left(0.01-1\right.$ ng. $\left.\mathrm{ml}^{-1}\right)$ inhibit IGF-I-induced protein synthesis [38, 39] and expression of the critical muscle differentiation factors, MyoD [38, $39]$ and myogenin [38, 39]. Extending these compelling data, we have previously demonstrated a dual role for IGF-I in C2 skeletal myoblasts in the presence of TNF- $\alpha$. It acts as a potent survival factor in the presence of higher dose TNF- $\alpha\left(10 \mathrm{ng} \cdot \mathrm{ml}^{-1}\right)$ but a potent apoptotic factor in the presence of non-myotoxic, low dose TNF- $\alpha\left(1.25\right.$ ng. $\left.l^{-1}\right)$ [1]. Our aim for the present study was to identify a critical gene using array technology for the insulin signalling pathway, whose expression profiles were upregulated in conditions of survival and downregulated under apoptotic conditions.

Of the insulin genes assessed Adra1d expression was appropriately altered as assessed using both array and qRT-PCR techniques. Particularly noticeable was its elevated expression in myoblasts administered with IGF-I (1.5 ng. ml $\left.{ }^{-1}\right)$. Myoblasts incubated with TNF- $\alpha$ alone (1.25 and $10 \mathrm{ng}^{-\mathrm{ml}^{-1}}$ ) resulted in a decrease in Adrald expression that was significant $(\mathrm{P}<0.05)$. With the addition of IGF-I to high dose TNF- $\alpha$, however, Adrald expression was brought to similar levels of control myoblasts that had been incubated with DM alone and consistent with survival. Potentially therefore IGF-I administration to high dose TNF- $\alpha$ may induce survival mechanisms that are linked to Adrald expression. Furthermore the death effect of low dose TNF- $\alpha /$ IGF-I was associated with decreased Adrald while its rescue with PD98059 was associated with increased expression of Adra1d, illustrating the pro-survival potential of this 
gene in myoblasts.

In relation to apoptosis, activation of the adrenergic receptors can induce the phosphorylation of protein kinase B (Akt) through a pathway that depends on PI(3)K activation [40]. Phosphorylated Akt mediates antiapoptosis through a number of transcriptionally-dependent (inhibition of FOXO activity thereby suppressing ubiquitin ligase expression) and post translational signalling pathways (activation of mTOR resulting in increase protein synthesis by modulating two distinct pathways, the p70S6K pathway and the Raptor-PHAS-1 pathway); phosphorylation of Glycogen-synthase kinase $3 \beta$ (GSK3 $\beta$ ), a kinase that blocks protein translation initiated by the eIF-2B protein $[5,41]$. It is possible that the $\mathrm{PI}(3) \mathrm{K}$ signalling is key to lowering apoptosis rates observed with high dose TNF- $\alpha$ and IGF-I co-incubation. Indeed, inhibition of the PI(3)K pathway using LY204002 (5 $\mu \mathrm{M})$ increased the incidence of myoblast death (data not shown) and significantly lowered Adrald expression across all culture conditions. Interestingly, where MAPK inhibition (PD98059 $(20 \mu \mathrm{M}))$ rescues the induction of death elicited by co-incubation of low dose TNF- $\alpha(1.25$ ng. $\mathrm{ml}^{-1}$ ) and IGF-I, addition, Adrald expression returned to baseline DM control levels, again associated with a potent survival effect and suggesting an inverse interaction between MAPK and Adrald expression. More detailed inhibitor studies downstream of the MAPK and PI(3)K pathways are required to ascertain the differential regulators of Adrald expression in situations of death or survival, respectively.

\section{Adrald inhibition in myoblasts}

Based on previous literature and findings from our gene array/qRT-PCR data it was hypothesised that inhibiting the expression of Adrald would lead to myoblast death. Since previous studies suggest that Adrald is associated with survival mechanisms (described above) gene silencing studies under conditions normally associated with myoblast survival in our experiments, were performed. Indeed, in agreement with our hypothesis we demonstrated that Adrald inhibition resulted in elevated death. Specifically the effects of Adrald inhibition were assessed in myoblasts administered with DM alone and IGF-I (1.5 ng. $\left.\mathrm{ml}^{-1}\right)$ alone where basal levels of cell death are observed under control, non-inhibited conditions. In addition, co-incubation of low dose TNF- $\alpha$ (1.25 ng. $\left.\mathrm{ml}^{-1}\right) /$ IGF-I with PD98059 $(20 \mu \mathrm{M})$, which blocks the apoptotic potential of the co-incubated growth factor and cytokine, was also assessed. Cell death was elevated significantly in the presence of Adrald siRNA to $\sim 30 \pm 5 \%$ at 48 -h and $100 \%$ at $72-h$. Differentiation, not surprisingly was also blocked, suggesting Adrald is essential in regulating pro-survival mechanisms and therefore normal myoblast function even under basal conditions.

Thus inhibiting $\alpha$-AR activity by gene silencing may subsequently down-regulate pro-survival signalling mechanisms such as via the PI(3)K pathway discussed above. Numerous additional mediators have been shown or implicated in $\alpha_{1}$-AR signalling including various ion channels, transporters, protein kinases and transcription factors and proteins related to cell cycle control. Consequently, inhibiting $\alpha$-AR stimulation potentially disrupts the function of multiple signalling pathways and this cumulative effect may explain the extensive levels of death and block on differentiation observed in these studies.

Our findings suggest that inhibition of Adrald leads to an increase in myoblast death in conditions that are generally associated with myoblast survival. This indicates Adra1d expression to be essential for the maintenance of myoblasts. This may be due to the multiple signalling pathways which the $\alpha_{1}$-ARs regulate which include the MAPK (inhibition results in survival and Adrald expression) and PI(3)K-Akt (inhibition results in death and Adra1d suppression) pathways that are associated with growth, differentiation, survival and metabolism

\section{Abbreviations}

Tumour necrosis factor-alpha (TNF- $\alpha$ ); Insulin-like growth factor-I (IGF-I); Adrenergic receptor alpha 1d (Adra1d); $\alpha_{1}$-Adrenergic receptors (AR).

\section{References}

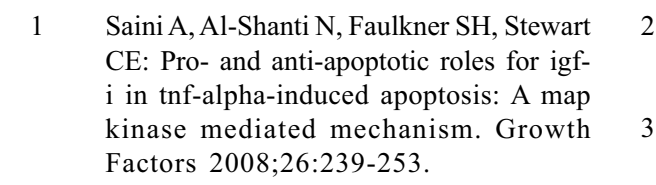

Morley JE, Baumgartner RN: Cytokine- $>4$ related aging process. J Gerontol A Biol Sci Med Sci 2004;59:M924-929.

Tisdale MJ: Cachexia in cancer patients. Nat Rev Cancer 2002;2:862-871.
Walsmith J, Roubenoff R: Cachexia in rheumatoid arthritis. Int $\mathrm{J}$ Cardiol 2002;85:89-99. 
Glass DJ: Signalling pathways that mediate skeletal muscle hypertrophy and atrophy. Nat Cell Biol 2003;5:87-90.

-6 Argiles JM, Lopez-Soriano FJ: The role of cytokines in cancer cachexia. Med Res Rev 1999;19:223-248.

7 Foulstone EJ, Meadows KA, Holly JM, Stewart CE: Insulin-like growth factors (igf-i and igf-ii) inhibit c2 skeletal myoblast differentiation and enhance tnf alpha-induced apoptosis. J Cell Physiol 2001;189:207-215.

$\checkmark 8$ Meadows KA, Holly JM, Stewart CE: Tumor necrosis factor-alpha-induced apoptosis is associated with suppression of insulin-like growth factor binding protein-5 secretion in differentiating murine skeletal myoblasts. J Cell Physiol 2000;183:330-337.

>9 Cooper RN, Tajbakhsh S, Mouly V, Cossu G, Buckingham M, Butler-Browne GS: In vivo satellite cell activation via myf5 and myod in regenerating mouse skeletal muscle. J Cell Sci 1999;112:2895-2901.

$>10$ Florini JR, Magri KA, Ewton DZ, James PL, Grindstaff K, Rotwein PS: "Spontaneous" Differentiation of skeletal myoblasts is dependent upon autocrine secretion of insulin-like growth factor-ii. J Biol Chem 1991;266:1591715923.

11 Stewart CE, Rotwein P: Growth, differentiation, and survival: Multiple physiological functions for insulin-like growth factors. Physiol Rev 1996;76:1005-1026.

12 Li Y, Jiang B, Ensign WY, Vogt PK, Han $\mathrm{J}$ : Myogenic differentiation requires signalling through both phosphatidylinositol 3-kinase and p38 map kinase. Cell Signal 2000;12:751-757.

13 Al-Shanti N, Stewart CE: Pd98059 enhances c2 myoblast differentiation through p38 mapk activation: A novel role for pd98059. J Endocrinol 2008;198:243-252.

14 Kyriakis JM, Avruch J: Mammalian mitogen-activated protein kinase signal transduction pathways activated by stress and inflammation. Physiol Rev 2001;81:807-869.

$>15$ Foulstone EJ, Huser C, Crown AL, Holly JM, Stewart CE: Differential signalling mechanisms predisposing primary human skeletal muscle cells to altered proliferation and differentiation: Roles of igf$i$ and tnfalpha. Exp Cell Res 2004;294:223-235.

> 16 Hubbard KB, Hepler JR: Cell signalling diversity of the gqalpha family of heterotrimeric g proteins. Cell Signal 2006;18:135-150.

17 Offermanns S: G-proteins as transducers $>29$ in transmembrane signalling. Prog Biophys Mol Biol 2003;83:101-130.

28
18 Exton JH: Regulation of phosphoinositide phospholipases by hormones, neurotransmitters, and other agonists linked to g proteins. Annu Rev Pharmacol Toxicol 1996;36:481-509.

19 Wu D, Katz A, Lee CH, Simon MI: Activation of phospholipase c by alpha 1 -adrenergic receptors is mediated by the alpha subunits of gq family. J Biol Chem 1992;267:25798-25802.

-20 Fain JN, Garcia-Sainz JA: Role of phosphatidylinositol turnover in alpha 1 and of adenylate cyclase inhibition in alpha 2 effects of catecholamines. Life Sci 1980;26:1183-1194.

21 Theroux TL, Esbenshade TA, Peavy RD, Minneman KP: Coupling efficiencies of human alpha 1-adrenergic receptor subtypes: Titration of receptor density and responsiveness with inducible and repressible expression vectors. Mol Pharmacol 1996;50:1376-1387.

22 Strosberg AD: Structure, function, and regulation of adrenergic receptors. Protein Sci 1993;2:1198-1209.

23 Xing M, Insel PA: Protein kinase cdependent activation of cytosolic phospholipase a2 and mitogen-activated protein kinase by alpha 1-adrenergic receptors in madin-darby canine kidney cells. J Clin Invest 1996;97:1302-1310.

24 Balboa MA, Insel PA: Stimulation of phospholipase d via alphal-adrenergic receptors in madin-darby canine kidney cells is independent of pkcalpha and epsilon activation. Molecular pharmacology 1998;53:221-227.

25 Parmentier JH, Ahmed A, Ruan Y, Gandhi GK, Saeed AE, Malik KU: Calcium and protein kinase c (pkc)-related kinase mediate alpha 1a-adrenergic receptorstimulated activation of phospholipase 38 $\mathrm{d}$ in rat-1 cells, independent of pkc. $J$ Pharmacol Exp Ther 2002;303:12061215.

26 Ruan Y, Kan H, Parmentier JH, Fatima S, Allen LF, Malik KU: Alpha-1a adrenergic receptor stimulation with phenylephrine promotes arachidonic acid release by activation of phospholipase $d$ in rat-1 fibroblasts: Inhibition by protein kinase a. J Pharmacol Exp Ther 1998;284:576-585.

27 Morgan NG, Charest R, Blackmore PF, Exton JH: Potentiation of alpha 1adrenergic responses in rat liver by a camp-dependent mechanism. Proc Natl Acad Sci U S A 1984;81:4208-4212. Yaffe D, Saxel O: Serial passaging and 40 differentiation of myogenic cells isolated from dystrophic mouse muscle. Nature 1977;270:725-727.

Elbashir SM, Harborth J, Lendeckel W, Yalcin A, Weber K, Tuschl T: Duplexes of 21-nucleotide rnas mediate rna interference in cultured mammalian cells. Nature 2001;411:494-498.
Elbashir SM, Lendeckel W, Tuschl T: Rna interference is mediated by 21 - and 22nucleotide rnas. Genes Dev 2001;15:188200 .

Elbashir SM, Martinez J, Patkaniowska A, Lendeckel W, Tuschl T: Functional anatomy of sirnas for mediating efficient rnai in drosophila melanogaster embryo lysate. Embo J 2001;20:6877-6888.

\$2 Tuschl T: Rna interference and small interfering rnas. Chembiochem 2001;2:239-245.

-33 Stewart CE, Newcomb PV, Savage PB, Dickens M, Tavare J, Holly JM: Increased, not decreased activation of the insulinlike growth factor (igf) receptor signalling pathway during ceramide-induced apoptosis. Growth Horm IGF Res 1999;9:131-142.

34 Zhao SP, Zeng LH: Elevated plasma levels of tumor necrosis factor in chronic heart failure with cachexia. International journal of cardiology 1997;58:257-261. Li YP, Schwartz RJ, Waddell ID, Holloway BR, Reid MB: Skeletal muscle myocytes undergo protein loss and reactive oxygen-mediated nf-kappab activation in response to tumor necrosis factor alpha. Faseb J 1998;12:871-880. Hoshino E, Pichard C, Greenwood CE, Kuo GC, Cameron RG, Kurian R, Kearns JP, Allard JP, Jeejeebhoy KN: Body composition and metabolic rate in rat during a continuous infusion of cachectin. Am J Physiol 1991;260:E27-36.

Lang CH, Frost RA, Nairn AC, MacLean DA, Vary TC: Tnf-alpha impairs heart and skeletal muscle protein synthesis by altering translation initiation. Am J Physiol Endocrinol Metab 2002;282:E336-347.

Broussard SR, McCusker RH, Novakofski JE, Strle K, Shen WH, Johnson RW, Freund GG, Dantzer R, Kelley KW: Cytokine-hormone interactions: Tumor necrosis factor alpha impairs biologic activity and downstream activation signals of the insulin-like growth factor $i$ receptor in myoblasts. Endocrinology 2003;144:2988-2996.

\39 Strle K, Broussard SR, McCusker RH, Shen WH, Johnson RW, Freund GG, Dantzer R, Kelley KW: Proinflammatory cytokine impairment of insulinlike growth factor i-induced protein synthesis in skeletal muscle myoblasts requires ceramide. Endocrinology 2004;145:4592-4602.

Bommakanti RK, Vinayak S, Simonds WF: Dual regulation of akt/protein kinase $b$ by heterotrimeric $g$ protein subunits. J Biol Chem 2000;275:3887038876.

Glass DJ: Molecular mechanisms modulating muscle mass. Trends $\mathrm{Mol}$ Med 2003;9:344-350. 